\title{
Role of inflammatory and liver function markers in assessing the prognosis of patients with COVID-19
}

\author{
SWATHY MOORTHY ${ }^{1}$, TEENA KOSHY ${ }^{2}$, MAHESH KUMAR K ${ }^{3}$ and SANTHI SILAMBANAN ${ }^{4}$ \\ Departments of ${ }^{1}$ General Medicine, and ${ }^{2}$ Human Genetics, Sri Ramachandra Institute of Higher Education and Research, \\ Chennai, Tamil Nadu 600116; ${ }^{3}$ Department of Physiology and Biochemistry, Government Yoga and \\ Naturopathy Medical College and Hospital, Chennai, Tamil Nadu 600106; ${ }^{4}$ Department of Biochemistry, \\ Sri Ramachandra Institute of Higher Education and Research, Chennai, Tamil Nadu 600116, India
}

Received June 8, 2021; Accepted August 23, 2021

DOI: $10.3892 /$ wasj.2021.123

\begin{abstract}
Severe acute respiratory syndrome coronavirus 2 (SARS-CoV-2) caused Coronavirus disease 2019 (COVID-19) in early December, 2019. The disease begins as a respiratory disease with varied outcomes, from complete recovery to long-standing complications, such as respiratory distress, heart ailments and stroke. The present study was undertaken to identify the derangements in liver function and inflammatory status which may be used to diagnose the severity and thus assess the prognosis of patients with COVID-19 infection. The present study was conducted at a tertiary care teaching hospital. The study population included 456 patients with COVID-19, confirmed by the reverse transcription-PCR of nasopharyngeal swabs for SARS-CoV-2. Data were collected from patient files. The study was approved by the institutional ethics committee. Data are expressed based on the normality of distribution. Variables were compared and correlation analyses were performed. Receiver operating characteristics and the Youden index were applied to obtain cut-off values. The results revealed an increase in C-reactive protein (CRP), ferritin, D-dimer, lactate dehydrogenase (LDH), aspartate aminotransferase (AST) and total bilirubin levels. In addition, there was evidence of leukocytosis, neutrophilia, lymphopenia and an increased neutrophil-to-lymphocyte ratio (NLR). The NLR exhibited an area under the curve of 0.77 , with a cut-off value of $2.23 \mathrm{mg} / \mathrm{l}$. Collectively, the findings of the present study demonstrate that the CRP, LDH, neutrophil and eosinophil
\end{abstract}

Correspondence to: Dr Santhi Silambanan, Department of Biochemistry, Sri Ramachandra Institute of Higher Education and Research, 1 Mount Poonamallee Road, Ramachandra Nagar, Porur, Chennai, Tamil Nadu 600116, India

E-mail: santhisilambanan@sriramachandra.edu.in

Key words: SARS-CoV-2, COVID-19, inflammation, lymphopenia, neutrophilia, De Ritis ratio, neutrophil-to-lymphocyte ratio counts along with the total leucocyte counts and absolute lymphocyte counts, NLR, erythrocyte sedimentation rate, D-dimer, ferritin and AST levels serve to predict the severity and prognosis of patients with COVID-19. AST appears to be a more sensitive marker than alanine aminotransferase. However, the De Ritis ratio, although not as sensitive as AST, may provide insight towards morbidity.

\section{Introduction}

Coronavirus disease 2019 (COVID-19) is caused by severe acute respiratory syndrome coronavirus 2 (SARS-CoV-2). The disease began in Wuhan, the capital of Hubei Province in China and soon developed into a global pandemic on March 11,2020 . The measures taken to prevent or contain the disease were not successful due to the rapidity of spread within the individual, as well as within the community (1). The incubation period of the virus is between 2 days to 2 weeks, during which time, the patient is asymptomatic, yet infectious. By the time the patients present with symptoms, the severity of the disease can be mild to moderate, depending on the presence of metabolic disorders, such as obesity, diabetes mellitus, hypertension and chronic obstructive pulmonary disease, amongst others (2).

When the virus infects the host, a battery of inflammatory responses is initiated to combat the pathogen. The inflammatory response is not terminated with scavenging the virus alone; it also damages tissues surrounding the area infected by the viral pathogen, as well as organs remote from the place of origin of infection. Thus, COVID-19-infected individuals tend to have systemic disease with the involvement of various organs, such as the heart, liver, central nervous system, blood vessels and lungs. The estimation of the extent of injury to these organs may guide clinicians as regards the extent or severity of the disease. Based on these features, the appropriate disease management can be initiated at the appropriate time (1). The present retrospective study was undertaken to identify the derangements in liver function and inflammatory biomarkers, which may be used to diagnose the severity and thus assess the prognosis of patients with COVID-19 infections. 


\section{Patients and methods}

Study design and setting. The present study was a retrospective study performed at the tertiary care teaching hospital, Sri Ramachandra Institute of Higher Education and Research in Chennai, India. The study population included patients with COVID-19, between June and August, 2020, aged $>18$ years, which was confirmed by reverse transcription-PCR of nasopharyngeal swabs for SARS-CoV-2 (assessed at one of the Indian Council of Medical Research-approved laboratories for RNA virus nucleic acid amplification tests). Patients discharged against medical advice for whom outcomes were not known were excluded from the study.

Classification of study population. The division of the patients into groups was based on peripheral oxygen saturation, respiratory rate and CT scan findings. The study population was subdivided into three groups as mild, moderate and severe based on peripheral oxygen saturation and respiratory rate. Mild, moderate and severe cases had an oxygen saturation of $\geq 94,91-93$ and $\leq 90 \%$, respectively and respiratory rates of $\leq 23,24-29$ and $\geq 30$ breaths $/ \mathrm{min}$, respectively (3). A CT scan of the chest was also used for grading the patients. According Bernheim et al (4), the degree of involvement of each lobe of the lungs was assessed and classified as none, minimal, mild, moderate and severe with the involvement of 0 (score 0 ), $1-25 \%$ (score 1), 26-50\% (score 2), $51-75 \%$ (score 3) and $76-100 \%$ (score 4) of lobe involvement, respectively. The sum of involvement of the five lobes of the lungs was presented as scores from 0 to 20 as the total severity score. The sum of the lobar scores indicated the overall severity: Total score (numerical) of $\leq 7$, mild; $8-17$, moderate; $\geq 18$, severe (4). A patient with a body temperature $>100^{\circ} \mathrm{F}$ was considered as febrile. A prolonged hospital duration was considered if the hospital stay was $>10$ days.

Data collection. Data collection was carried out by the manual perusal of inpatient case sheets, investigations of computerized patient data systems and the transcription database for discharge summaries. Details of basic demographic characteristics, and information for symptoms, such as fever, throat pain, cough, dyspnea, diarrhea, anosmia and myalgia, hemodynamic parameters upon admission and during the course of stay, daily clinical assessment, initial laboratory tests, such as complete blood count, liver function tests [bilirubin, aspartate aminotransferase (AST), alanine aminotransferase (ALT), alkaline phosphatase, albumin, C-reactive protein (CRP)] and the detection of serum ferritin, lactate dehydrogenase (LDH) and D-dimer levels were collected. The laboratory parameters were analyzed using standard accepted methods in the National Accreditation Board for Testing and Calibration Laboratories (NABL) accredited Sri Ramachandra Laboratory services. In addition, information regarding chest imaging, medications administered (steroids, heparin, remdesivir and antibiotics), oxygen support, organ dysfunction, secondary sepsis and final outcome were collected and deidentified for personal details prior to the analysis. The primary outcomes of interest in the study were clinical severity, elevated inflammatory marker levels, high D-dimer levels, a prolonged hospital duration and the condition of the patients at discharge.
Ethics statement. The present study was approved by the Institutional Ethics Committee of Sri Ramachandra Institute of Higher Education and Research, with a waiver of informed consent of patients, as permitted by the national regulatory body (IEC-NI/20/AUG/75/49, dated 08-08-2020). Patient identification was de-identified reversibly for the purpose of the analysis.

Statistical analysis. Normality distribution was evaluated using the the Kolmogorov-Smirnov test for all variables. Depending on the presence or absence of the normality of distribution, data are expressed as the mean \pm standard deviation or the median and interquartile range (IQR). Between-group differences of variables were compared using one-way ANOVA followed by Tukey's post hoc test. The association between variables was obtained by Pearson's correlation analysis. Receiver operating characteristics (ROC) curve analysis was performed to estimate the area under the curve (AUC) with the 95\% confidence interval (CI) and the cut-off point according to the Youden index. All statistical analyses were conducted using R statistical software version 4.0.2 (The R Project for Statistical Computing; https://www.r-project.org/). $P<0.05$ was considered to indicate a statistically significant difference.

\section{Results}

In the present study, 456 patients with confirmed COVID-19 infection (277 males and 179 females) were included in the analysis. The median age of the patients was 54 years (IQR, 41-68). Of the 456 patients, 324 patients (71\%) had mild, 66 patients (14.5\%) had moderate and 66 patients (14.5\%) had severe COVID-19 infection. The baseline demographic and laboratory parameters of the study population are presented in Table I. The most common presenting clinical features were fever, sore throat, cough, dyspnea, diarrhea, anosmia and myalgia in the order of occurrence. The patients in the younger age group with an age of $48.85 \pm 14.50$ years recovered completely. Leukocytosis with progressive neutrophilia and lymphopenia, and an increased neutrophil-to-lymphocyte ratio (NLR) were noted in patients with mild to severe disease. The concentrations of liver function markers in the three study groups are presented in Table II. There was highly significant increase in AST, ALT and total bilirubin levels from the mild to severe disease groups. The ROC curve of hematological markers is illustrated in Fig. 1. The AUC was found to be high for the NLR, eosinophil count and D-dimer levels. The ROC curves of various inflammatory and liver function markers are presented in Fig. 2. The AUC was found to be high for the CRP and LDH levels, and the neutrophil count. The AUC of the various hematological, inflammatory and liver function markers among the three study groups are presented in Table III. According to the AUC values, the studied biomarkers were categorized into four groups for predicting the severity and prognosis of COVID-19 infection as follows: i) AUC 1.0-0.8, excellent diagnostic accuracy (CRP, LDH and neutrophil counts); ii) AUC 0.8-0.6, very good diagnostic accuracy [NLR, eosinophil count, erythrocyte sedimentation rate (ESR), D-dimer, ferritin, total white blood cell (WBC) counts, absolute lymphocyte counts (ALC) and AST]; iii) AUC 0.6-0.5, good diagnostic accuracy (De Ritis and ALT); and 
Table I. Levels of the inflammatory markers of the study participants.

\begin{tabular}{|c|c|c|c|c|c|}
\hline Parameters & $\begin{array}{l}\text { Mild } n=324 \\
\quad(72 \%)\end{array}$ & $\begin{array}{c}\text { Moderate } \\
n=66(14 \%)\end{array}$ & $\begin{array}{c}\text { Severe } n=66 \\
(14 \%)\end{array}$ & $\begin{array}{l}\text { ANOVA } \\
\text { P-value }\end{array}$ & $\begin{array}{l}\text { Tukey's post hoc } \\
\text { analysis: P-value }\end{array}$ \\
\hline Age (years) & $48.85 \pm 14.50$ & $56.63 \pm 11.5$ & $61.47 \pm 11.03$ & $<0.001$ & $\begin{array}{c}\text { Mild-moderate }=0.01 \\
\text { Mild-severe }=0.002 \\
\text { Moderate-severe }=0.05\end{array}$ \\
\hline $\operatorname{Sex}(M / F)(n, \%)$ & $\begin{array}{c}188 / 136 \\
(58 / 42 \%)\end{array}$ & $\begin{array}{c}45 / 21 \\
(68 / 32 \%)\end{array}$ & $44 / 22(67 / 33 \%)$ & 0.04 & - \\
\hline Length of hospital stay (days) & $5.1 \pm 2.8$ & $8.5 \pm 3.2$ & $17.6 \pm 5.2$ & $<0.00001$ & $\begin{array}{c}\text { Mild-moderate }=0.00001 \\
\text { Mild-severe }=0.00001 \\
\text { Moderate-severe }=0.00001\end{array}$ \\
\hline $\mathrm{Hb}(\mathrm{g} \%)^{\mathrm{a}}$ & $12.92 \pm 1.72$ & $12.42 \pm 1.79$ & $12.35 \pm 2.00$ & 0.06 & - \\
\hline Total WBC count $\left(\text { cells } / \mathrm{mm}^{3}\right)^{\mathrm{b}}$ & $\begin{array}{c}5,900 \\
(2,000-15,800)\end{array}$ & $\begin{array}{c}6,700 \\
(2,500-14,700)\end{array}$ & $\begin{array}{c}8,961 \\
(3,800-43,000)\end{array}$ & $<0.001$ & $\begin{array}{c}\text { Mild-moderate }=0.074 \\
\text { Mild-severe }=0.001 \\
\text { Moderate-severe }=0.002\end{array}$ \\
\hline Neutrophil count $(\%)^{\mathrm{a}}$ & $58.29 \pm 12.66$ & $72.35 \pm 12.69$ & $77.30 \pm 11.54$ & $<0.001$ & $\begin{array}{c}\text { Mild-moderate }=0.006 \\
\text { Mild-severe }=0.002 \\
\text { Moderate-severe }=0.07\end{array}$ \\
\hline Lymphocyte count $(\%)^{\mathrm{b}}$ & $30.11(2.3-61.0)$ & $18.35(2.5-41.2)$ & $14.30(1.4-38.4)$ & $<0.001$ & $\begin{array}{c}\text { Mild-moderate }=0.0001 \\
\text { Mild-severe }=0.002 \\
\text { Moderate-severe }=0.086\end{array}$ \\
\hline Monocyte count $(\%)^{\mathrm{a}}$ & $9.38 \pm 3.13$ & $7.71 \pm 3.51$ & $7.12 \pm 3.69$ & $<0.001$ & $\begin{array}{c}\text { Mild-moderate }=0.01 \\
\text { Mild-severe }=0.001 \\
\text { Moderate-severe }=0.56\end{array}$ \\
\hline Eosinophil count $(\%)^{\mathrm{b}}$ & $0.81(0-34)$ & $0.25(0-6.1)$ & $0.11(0-3.9)$ & 0.002 & $\begin{array}{c}\text { Mild-moderate }=0.02 \\
\text { Mild-severe }=0.006 \\
\text { Moderate-severe }=0.89\end{array}$ \\
\hline $\mathrm{NLR}^{\mathrm{b}}$ & $\begin{array}{c}1.94 \\
(0.23-26.91)\end{array}$ & $\begin{array}{c}3.96 \\
(1.06-35.08)\end{array}$ & $\begin{array}{c}5.36 \\
(1.22-68.36)\end{array}$ & $<0.001$ & $\begin{array}{c}\text { Mild-moderate }=0.001 \\
\text { Mild-severe }=0.001 \\
\text { Moderate-severe }=0.06\end{array}$ \\
\hline $\operatorname{ALC}\left(\text { cells } / \mathrm{mm}^{3}\right)^{\mathrm{b}}$ & $\begin{array}{c}1,694 \\
(312-5,707)\end{array}$ & $\begin{array}{c}1,084 \\
(345-3,321)\end{array}$ & $\begin{array}{c}1,016 \\
(215-2,656)\end{array}$ & $<0.001$ & $\begin{array}{c}\text { Mild-moderate }=0.0001 \\
\text { Mild-severe }=0.001 \\
\text { Moderate-severe }=0.99\end{array}$ \\
\hline $\operatorname{ESR}(\mathrm{mm} / \mathrm{h})^{\mathrm{b}}$ & $12(1-95)$ & $36(7-87)$ & $37(5-53)$ & 0.02 & $\begin{array}{c}\text { Mild-moderate }=0.001 \\
\text { Mild-severe }=0.24 \\
\text { Moderate-severe }=0.10\end{array}$ \\
\hline $\mathrm{CRP}(\mathrm{mg} / \mathrm{l})^{\mathrm{b}}$ & $0.80(0.1-75)$ & $7.35(0.2-46.5)$ & $6.90(0.3-36.6)$ & $<0.001$ & $\begin{array}{c}\text { Mild-moderate }=0.001 \\
\text { Mild-severe }=0.001 \\
\text { Moderate-severe }=0.68\end{array}$ \\
\hline Ferritin $(\mathrm{ng} / \mathrm{ml})^{\mathrm{b}}$ & $\begin{array}{c}103.6 \\
(4.3-4,820.0)\end{array}$ & $\begin{array}{c}239.0 \\
(14.5-1,683.0)\end{array}$ & $\begin{array}{c}303.9 \\
{[28.8-7,500.0]}\end{array}$ & 0.0001 & $\begin{array}{c}\text { Mild-moderate }=0.02 \\
\text { Mild-severe }=0.001 \\
\text { Moderate-severe }=0.01\end{array}$ \\
\hline $\mathrm{D}$-dimer $(\mathrm{mg} / \mathrm{l})^{\mathrm{b}}$ & $\begin{array}{c}0.40 \\
(0.01-21.00)\end{array}$ & $\begin{array}{c}0.81 \\
(0.12-4.95)\end{array}$ & $\begin{array}{c}0.89 \\
(0.23-59.64)\end{array}$ & $<0.001$ & $\begin{array}{c}\text { Mild-moderate }=0.51 \\
\text { Mild-severe }=0.001 \\
\text { Moderate-severe }=0.001\end{array}$ \\
\hline $\mathrm{LDH}(\mathrm{U} / \mathrm{l})^{\mathrm{b}}$ & $225(18-946)$ & $324(117-645)$ & $411(199-978)$ & $<0.001$ & $\begin{array}{c}\text { Mild-moderate }=0.001 \\
\text { Mild-severe }=0.002 \\
\text { Moderate-severe }=0.001\end{array}$ \\
\hline
\end{tabular}

avalues of mild, moderate and severe are expressed as the mean $\pm \mathrm{SD}$; ${ }^{\mathrm{b}}$ values of mild, moderate and severe are expressed as the median and range. $\mathrm{Hb}$, hemoglobin; WBC, white blood cell; NLR, neutrophil-to-lymphocyte ratio; ALC, absolute lymphocyte count; ESR, erythrocyte sedimentation rate; CRP, C-reactive protein, $\mathrm{LDH}$, lactate dehydrogenase.

iv) AUC $<0.5$, poor diagnostic accuracy (lymphocyte differential counts and monocyte differential counts).
The results of the correlation analysis between the De Ritis ratio and other markers of liver function and inflammatory 
Table II. Levels of liver function parameters in the study participants.

\begin{tabular}{|c|c|c|c|c|c|}
\hline Parameters & Mild n=324 & Moderate $n=66$ & Severe $n=66$ & P-value & Tukey's post hoc analysis P-value \\
\hline $\operatorname{AST}(\mathrm{U} / 1)^{\mathrm{a}}$ & $29(14-159)$ & $37(3-288)$ & $47(25-143)$ & 0.006 & $\begin{array}{c}\text { Mild-moderate }=0.008 \\
\text { Mild-severe }=0.11 \\
\text { Moderate-severe }=0.82\end{array}$ \\
\hline $\operatorname{ALT}(\mathrm{U} / \mathrm{l})^{\mathrm{a}}$ & $26(8-128)$ & $29(10-313)$ & $73(20-148)$ & 0.003 & $\begin{array}{c}\text { Mild-moderate }=0.17 \\
\text { Mild-severe }=0.02 \\
\text { Moderate-severe }=0.20\end{array}$ \\
\hline De Rittis ratio ${ }^{a}$ & $1.21(0.45-3.13)$ & $1.31(0.08-5.54)$ & $0.97(0.45-2.35)$ & 0.56 & - \\
\hline Serum albumin $(\mathrm{g} / \mathrm{dl})^{\mathrm{b}}$ & $4.14 \pm 0.42$ & $3.70 \pm 0.37$ & $3.64 \pm 0.57$ & 0.09 & $\begin{array}{c}\text { Mild-moderate }=0.001 \\
\text { Mild-severe }=0.001 \\
\text { Moderate-severe }=0.99\end{array}$ \\
\hline Total bilirubin $(\mathrm{mg} / \mathrm{dl})^{\mathrm{a}}$ & $0.54(0.19-2.30)$ & $0.61(0.19-12.83)$ & $0.83(0.3-3.1)$ & 0.008 & $\begin{array}{c}\text { Mild-moderate }=0.04 \\
\text { Mild-severe }=0.28 \\
\text { Moderate-severe }=0.91\end{array}$ \\
\hline Direct bilirubin $(\mathrm{mg} / \mathrm{dl})^{\mathrm{a}}$ & $0.11(0.04-9.0)$ & $0.17(0.06-6.62)$ & $0.18(0.05-1.78)$ & 0.74 & - \\
\hline
\end{tabular}

${ }^{a}$ Values of mild, moderate and severe are expressed as median and range; ${ }^{b}$ Values of mild, moderate and severe are expressed as the mean $\pm \mathrm{SD}$. AST, aspartate transaminase; ALT, alanine transaminase.

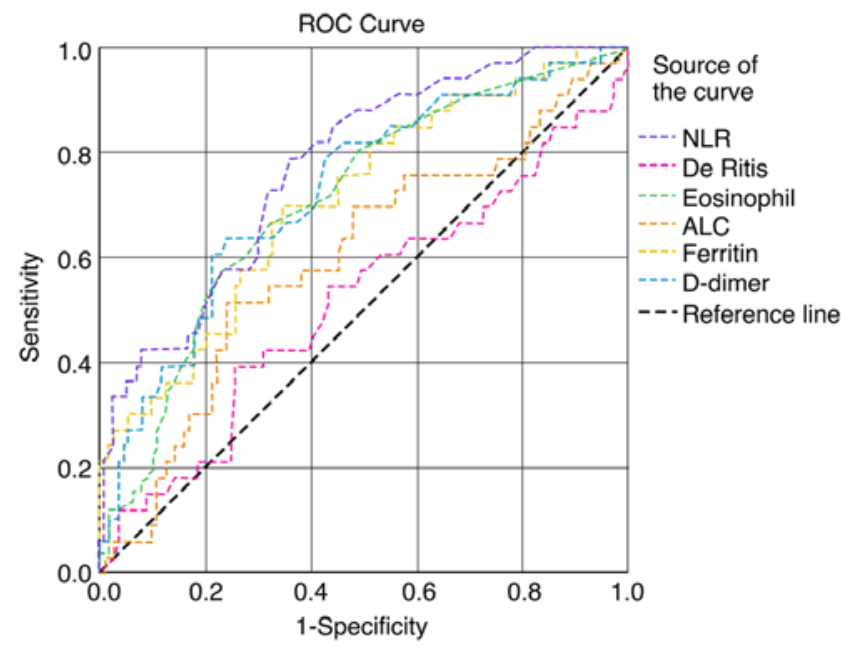

Figure 1. Receiver operating characteristics curves of eosinophil counts, ALC, NLR, D-dimer levels, ferritin levels and the De Ritis ratio. ALC, absolute lymphocyte count; NLR, neutrophil-to-lymphocyte ratio.

markers are presented in Table IV. Of the liver function parameters examined, the De Ritis ratio exhibited positive correlations with total bilirubin and AST, and negative correlations with serum albumin and ALT. When compared with the inflammatory markers, the De Ritis ratio exhibited a positive correlation with D-dimer levels, and negative correlations with hemoglobin levels and the WBC count. The results of the correlation between the NLR and other inflammatory markers, as well as liver function markers are presented in Table V. NLR exhibited positive correlations with the neutrophil count, CRP, ferritin, D-dimer and LDH levels, and negative correlations with the lymphocyte count and monocyte count. When compared with liver function markers, NLR exhibited a positive correlation with ALT, and a negative correlation with serum albumin levels.

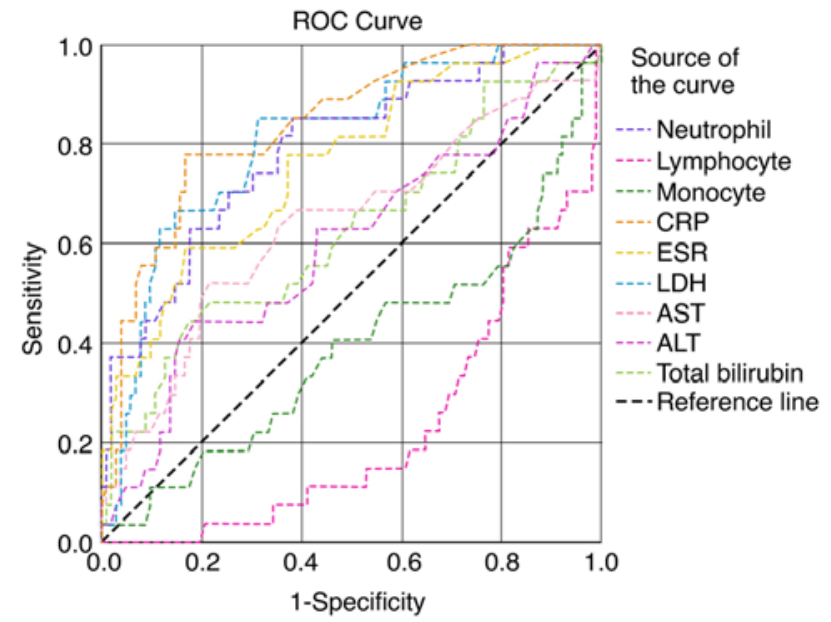

Figure 2. Receiver operating characteristics curves of neutrophil, lymphocyte and monocyte counts, and total WBC counts, ESR, CRP, LDH, AST and ALT levels. WBC, white blood cell; CRP, C-reactive protein; ESR, erythrocyte sedimentation rate; $\mathrm{LDH}$, lactate dehydrogenase; AST, aspartate aminotransferase; ALT, alanine aminotransferase.

\section{Discussion}

When COVID-19 infection progresses to severe disease, it has been found to present a systemic manifestation with multiorgan failure and septicemia, which manifests as acute respiratory distress syndrome, respiratory failure, acute liver injury, renal injury or failure and cardiovascular manifestations (5). Few patients exhibit an inadequate pro-inflammatory response, resulting in severe disease manifestations. This presentation is known as the cytokine response syndrome (CRS) or cytokine storm syndrome (CSS) $(1,6)$. The present study demonstrated that patients with severe disease were in the age group of $61.47 \pm 11.03$ years, during which the onset of comorbid conditions was observed. COVID-19 has predominantly affected 
Table III. AUC of the biochemical parameters of the study participants.

\begin{tabular}{|c|c|c|c|c|c|}
\hline Variables & AUC & SE & $95 \% \mathrm{CI}$ & Cut-off value & P-value \\
\hline Total WBC count & 0.64 & 0.043 & 0.56 to 0.72 & 5750 & 0.002 \\
\hline Neutrophil count & 0.828 & 0.032 & 0.76 to 0.89 & 65.4 & 0.0001 \\
\hline Lymphocyte count & 0.178 & 0.030 & 0.11 to 0.23 & - & 0.0001 \\
\hline Monocytes count & 0.38 & 0.052 & 0.28 to 0.49 & - & 0.01 \\
\hline Eosinophil count & 0.71 & 0.02 & 0.66 to 0.76 & 0.35 & 0.001 \\
\hline ALC & 0.60 & 0.05 & 0.48 to 0.71 & - & 0.08 \\
\hline NLR & 0.77 & 0.45 & 0.68 to 0.85 & 2.23 & 0.001 \\
\hline ESR & 0.74 & 0.041 & 0.66 to 0.82 & 22.5 & 0.001 \\
\hline D-dimer & 0.72 & 0.05 & 0.62 to 0.82 & 0.54 & 0.001 \\
\hline Ferritin & 0.71 & 0.05 & 0.61 to 0.81 & 173.60 & 0.001 \\
\hline CRP & 0.825 & 0.03 & 0.76 to 0.88 & 1.79 & 0.0001 \\
\hline LDH & 0.808 & 0.036 & 0.73 to 0.88 & 240.5 & 0.0001 \\
\hline AST & 0.63 & 0.07 & 0.49 to 0.77 & 71 & 0.04 \\
\hline ALT & 0.56 & 0.05 & 0.42 to 0.69 & - & 0.34 \\
\hline De Ritis ratio & 0.51 & 0.06 & 0.39 to 0.63 & - & 0.74 \\
\hline
\end{tabular}

Values in bold font indicate statistically significant differences $(\mathrm{P}<0.05)$. AUC, area under the curve; SE, standard error; $\mathrm{CI}$, confidence interval; WBC, white blood cell; NLR, neutrophil-to-lymphocyte ratio; ALC, absolute lymphocyte count; ESR, erythrocyte sedimentation rate; CRP, C-reactive protein, LDH, lactate dehydrogenase; AST, aspartate transaminase; ALT, alanine transaminase.

males compared with females; this may possibly be due to the increased prevalence of comorbid conditions in males. In addition, the number of males admitted to the hospital was markedly higher than the number of female patients, which may be a confounder. In the present study, the number of males affected with the mild, moderate and severe forms of the disease were 58, 68 and 67\%, respectively (Table I). The length of hospital stay was also proportionate to the severity of the disease, being $5.1 \pm 2.8,8.5 \pm 3.2$ and 17.6 \pm 5.2 days in the mild, moderate and severe groups, respectively (Table I).

According to the study by Ali et al (6), it was found that older individuals, particularly those with underlying co-morbidities had a higher risk of developing severe disease. Patients who developed severe disease required an extended hospital stay mostly in the intensive care unit (ICU) with mechanical ventilation (6). The clinical course of the disease is influenced by the age of the patient, the presence of comorbidities, the severity of symptoms and the lymphopenia level. Patients who require a longer hospital stay have been shown to have higher incidences of complications and secondary bacterial infections $(7,8)$. The median age of survivors and non-survivors has been shown to be 54 and 70 years, respectively (9). The median age of patients who experience severe outcomes is 65 years, and $75 \%$ of these patients are males (10). As previously demonstrated, a chest CT scan of patients with COVID-19 has demonstrated various patterns and degrees of involvement of the lungs. Male patients with severe COVID-19 infection have been shown to exhibit lower levels of testosterone and dihydrotestosterone than other male patients with COVID-19 infection; however, the role of these hormones remains unclear(11).

Patients with COVID-19 are assessed for multiorgan failure so that early ICU care with assisted mechanical ventilation may be initiated. Routine laboratory blood tests have been found to add value to radiological and clinical features (12). In the present study, serially increasing total WBC counts were observed among the COVID-19 cases and these increases were statistically significant, and were more pronounced between the moderate and severe cases $(\mathrm{P}=0.002$; Table I). The AUC for the WBC count was 0.64 with a $95 \%$ CI of $0.56-0.72$ with a cut-off of 5,750 cells $/ \mathrm{mm}^{3}$ (Fig. 2 and Table III). In addition, progressive lymphopenia was observed from mild to severe disease, particularly between the mild and moderate groups $(\mathrm{P}=0.0001$; Table I). Statistically significant decreases were observed in the monocyte $(\mathrm{P}=0.01)$ and eosinophil $(\mathrm{P}=0.02)$ counts between patients with mild and moderate COVID-19 infection (Table I). Widespread systemic inflammation has been shown to lead to thrombotic, vascular and ischemic injury with multiorgan damage (13). Hyperinflammation leads to the progression of disease to a severe form with severe outcomes (14). As per the metanalysis conducted by Zenga et al (15), the assessment of inflammatory markers may guide clinicians in making decisions regarding the severity of the disease.

Lymphopenia may be due to the direct injury of lymphocytes, resulting in the altered structure and/or function of lymphocytes. Thus, lymphopenia may be considered a hallmark of SARS-CoV-2 infection, even in mild cases of infection. The degree of lymphopenia may address the severity of the disease and the onset of complications (12). Interleukin-6 (IL-6) plays a role in the apoptosis of T-lymphocytes. The CSS-induced bone marrow suppression of lymphocyte production, as well as the overconsumption of lymphocytes subsequent to inflammatory reactions occur in the lungs $(11,13,16)$. Researchers have also documented the decrease in the eosinophil count (12). In the present study, there was gradual decrease in the eosinophil count from mild to severe disease. The combination of low 
Table IV. Correlation between De Ritis ratio and other parameters in patients with COVID-19.

\begin{tabular}{lcc}
\hline Parameters & Correlation coefficient (r) & P-value \\
\hline $\mathrm{Hb}$ & $\mathbf{- 0 . 4 2}$ & $<\mathbf{0 . 0 0 1}$ \\
Total WBC count & $\mathbf{- 0 . 2 3}$ & $\mathbf{0 . 0 0 3}$ \\
Neutrophil count & -0.14 & 0.08 \\
Lymphocyte count & 0.12 & 0.11 \\
Monocytes count & 0.14 & 0.07 \\
Eosinophil count & -0.009 & 0.91 \\
NLR & -0.15 & 0.06 \\
Serum albumin & -0.24 & $\mathbf{0 . 0 0 2}$ \\
ESR & 0.16 & $\mathbf{0 . 0 5}$ \\
CRP & -0.001 & 0.99 \\
Total bilirubin & 0.53 & $\mathbf{0 . 0 0 1}$ \\
Direct bilirubin & $\mathbf{- 0 . 1 8}$ & $\mathbf{0 . 8 4}$ \\
AST & $\mathbf{0 . 3 0}$ & $\mathbf{0 . 0 0 1}$ \\
ALT & $\mathbf{- 0 . 2 9}$ & $\mathbf{0 . 0 0 3}$ \\
Ferritin & -0.18 & $\mathbf{0 . 0 2}$ \\
D-dimer & $\mathbf{0 . 3 5}$ & $<\mathbf{0 . 0 0 1}$ \\
LDH & -0.01 & 0.88 \\
\hline
\end{tabular}

Values in bold font indicate statistically significant differences $(\mathrm{P}<0.05)$. Hb, hemoglobin; WBC, white blood cell; NLR, neutrophil-to-lymphocyte ratio; ALC, absolute lymphocyte count; ESR, erythrocyte sedimentation rate; CRP, C-reactive protein, $\mathrm{LDH}$, lactate dehydrogenase; AST, aspartate transaminase; ALT, alanine transaminase.

lymphocytes and eosinophils is a strong indicator of the presence of infection $(12,17)$.

The present study demonstrated a statistically significant increase in the neutrophil count $(\mathrm{P}=0.001)$, particularly between between mild and moderate cases $(\mathrm{P}=0.006)$. The AUC was 0.828 with a cut-off value of $65.4 \%$ (Fig. 2 and Table III). The NLR also steadily increased with the severity of infection $(\mathrm{P}<0.001)$, more so between the mild and moderate cases $(\mathrm{P}=0.001$; Table I). Lymphopenia along with neutrophilia represented by the NLR, was considered to be a better indicator of prognosis of COVID-19 infection. Studies have demonstrated that the WBC count, neutrophil absolute count, NLR and the platelet-to-lymphocyte ratio were significantly higher in patients in the ICU with severe disease $(7,18)$. According to Zhao et al (9), survivors and non-survivors had normal absolute neutrophil counts, although survivors had significantly lower neutrophil counts than non-survivors. Patients with moderate to severe infection with a WBC count $>10,000$ cells $/ \mathrm{mm}^{3}$ have been shown to require inpatient treatment (10). As previously demonstrated, the lower the lymphocyte count and the longer the duration of lymphopenia, the poorer the prognosis (19).

Macrophages have been found to play a central role both at the onset and during SARS-CoV-2 infection $(20,21)$. In the present study, the AUC for NLR was 0.77 with a $95 \%$ CI of 0.68-0.85 ( $\mathrm{P}=0.001)$ and a cut-off value of 2.23 (Fig. 1 and Table III). NLR also exhibited a positive correlation with the total WBC count, neutrophil count, and with the ALT, CRP, ferritin, D-dimer and LDH levels. The NLR exhibited a negative correlation with lymphocytes, monocytes and serum albumin levels (Table V). In addition, there was significant increase in the ESR $(\mathrm{P}=0.02$; Table I). The AUC was 0.74 with a $95 \%$ CI of $0.66-0.82(\mathrm{P}=0.001)$ and a cut-off value of $22.5 \mathrm{~mm} / \mathrm{h}$ (Fig. 2 and Table III). ESR can be used along with CRP levels as inflammatory markers to assess the outcomes of patients with COVID-19 infection (12). ESR levels have been found to exhibit a significant negative correlation with albumin levels. Increased fibrinogen in acute inflammatory conditions can cause an increased ESR. Albumin exerts a suppressive effect on ESR; hence, in COVID-19-infected patients, particularly those with severe infection, when the serum albumin levels decrease, the ESR tends to increase $(10,22)$.

Inflammation induces alveolar injury and hypoxemia activates the vascular endothelial response, which then augments thrombus formation (23). IL-6, through the Janus kinase (JAK)/signal transducer and activator of transcription (STAT) pathway leads to in increased transcription of acute phase proteins (24-26). The dysregulated immune response is characterized by a substantial reduction in peripheral lymphocyte counts in patients with COVID-19, and is associated with a high risk of developing secondary bacterial infections (27). In the present study, a significant progressive increase in CRP $(\mathrm{P}<0.001)$ was observed. The AUC for CRP was 0.825 with a $95 \%$ CI of 0.76-0.88 (Fig. 2 and Table III). In addition, the WBC count, and D-dimer, CRP and LDH levels were found to play a role in the assessment of the prognosis of patients with COVID-19. There was significant increase in D-dimer levels as the disease severity progressed $(\mathrm{P}<0.001$; Table I). The levels of CRP and other pro-inflammatory cytokines, chemokines and NLR have been shown to be increased in severe cases of COVID-19, possibly due to a hyper-inflammatory response in these individuals (28). CRP levels $>10 \mathrm{mg} / \mathrm{l}$ may aid in assessing the prognosis of patients with severe COVID-19 infection (29). In SARS-CoV-2 infection, the state of coagulopathy is presumed to be precipitated by the complex interaction between inflammatory and pro-thrombotic factors (30). Coronavirus causes endothelial inflammation and injury, resulting in disseminated intravascular coagulation. Patients with D-dimer levels $>1 \mathrm{mg} / \mathrm{l}$ have been shown to have an unfavorable disease progression $(6,12,13,31)$.

In the present study, ferritin levels exhibited a significant increase as the disease advanced to a severe form $(\mathrm{P}=0.0001$; Table I) The AUC for ferritin was $0.71(\mathrm{P}=0.001)$ with a cut-off value of $173.60 \mathrm{ng} / \mathrm{ml}$ (Fig. 1 and Table III). Ferritin, as a positive acute phase protein, is considered to be a marker of adverse outcomes in individuals infected with SARS-CoV-2. $(12,32)$. The present study also observed a significant increase in LDH levels $(\mathrm{P}<0.001$; Table I). The AUC for LDH was 0.808 $(\mathrm{P}=0.0001)$ with a cut-off value of $240.5 \mathrm{U} / 1$ (Fig. 2 and Table III). LDH is a non-specific marker of tissue damage. Studies have demonstrated that $\sim 50 \%$ of infected individuals have increased CRP, LDH and ferritin levels, and an increased ESR $(9,33,34)$. These alterations may be due to the severe systemic immune response characteristic feature of CSS (13). In patients with COVID-19, the blood levels of D-dimer and LDH have been shown to exhibit a strong positive association with mortality $(10,35)$. In patients with COVID-19, the NLR has been shown to exhibit a positive correlation with CRP, $\mathrm{LDH}$, ferritin, D-dimer and troponin-I levels; these variables 
Table V. Correlation between NLR and other parameters in patients with COVID-19.

\begin{tabular}{lcl}
\hline Parameters & Correlation coefficient (r) & P-value \\
\hline Hb & $\mathbf{- 0 . 1 7}$ & $\mathbf{0 . 0 0 0 2}$ \\
Total WBC count & $\mathbf{0 . 5 5}$ & $\mathbf{0 . 0 0 1}$ \\
Neutrophil count & 0.63 & $\mathbf{0 . 0 0 1}$ \\
Lymphocyte count & -0.62 & $\mathbf{0 . 0 0 1}$ \\
Monocytes count & -0.35 & $\mathbf{0 . 0 0 0 1}$ \\
Eosinophil count & -0.14 & 0.02 \\
De Ritis ratio & -0.15 & 0.06 \\
Serum Albumin & -0.26 & $\mathbf{0 . 0 0 1}$ \\
ESR & 0.17 & $\mathbf{0 . 0 0 7}$ \\
CRP & 0.39 & $\mathbf{0 . 0 0 1}$ \\
Total Bilirubin & 0.12 & 0.12 \\
Direct Bilirubin & -0.02 & 0.79 \\
AST & $\mathbf{0 . 1 3}$ & $\mathbf{0 . 0 9}$ \\
ALT & $\mathbf{0 . 2 4}$ & $\mathbf{0 . 0 0 2}$ \\
Ferritin & 0.23 & $\mathbf{0 . 0 0 1}$ \\
D-Dimer & $\mathbf{0 . 2 2}$ & $\mathbf{0 . 0 0 1}$ \\
LDH & 0.31 & 0.001 \\
\hline
\end{tabular}

Values in bold font indicate statistically significant differences $(\mathrm{P}<0.05)$. Hb, hemoglobin; WBC, white blood cell; NLR, neutrophil-to-lymphocyte ratio; ALC, absolute lymphocyte count; ESR, erythrocyte sedimentation rate; CRP, C-reactive protein, $\mathrm{LDH}$, lactate dehydrogenase; AST, aspartate transaminase; ALT, alanine transaminase.

may suggest the duration of hospital or ICU stay, as well as disease outcomes (7).

Patients with severe COVID-19 infection also exhibit a high incidence of liver involvement during their clinical course (20,36). COVID-19 may affect multiple organs due to disseminated intravascular coagulation (DIC), and hypoxia with hypoperfusion, leading to shock (37). Elevated levels of transaminases and bilirubin along with decreased albumin and prealbumin levels have also been found in patients with COVID-19 with suspected liver inflammation or injury. The involvement of the liver in COVID-19 is indicative of a poor outcome. In addition, certain drugs used in the treatment of COVID-19 have been found to aggravate liver damage (13). In the present study, the levels of AST exhibited a steady increase as the severity of the disease increased $(\mathrm{P}=0.006$; Table I). The AUC for AST was 0.63 with a cut-off value of 71 U/1 (Fig. 2 and Table III). The ALT levels also exhibited a statistically significant increase $(\mathrm{P}=0.003)$. The AUC for ALT was 0.56, which was lower than that of AST (Fig. 2 and Table III). The AST:ALT (De Ritis) ratio did not exhibit a statistically significant difference, unlike the results obtained from other studies (38-40). This may be due to the fact that in the present study, the distribution of study participants was not the same in the various groups: 72, 14 and $14 \%$ in mild, moderate and severe cases, respectively.

The De Ritis ratio is more likely to increase when the severity or chronicity of the disease is high. An altered De Ritis ratio has been found to be associated with hyperbilirubinemia in patients (38). Moderate to severe liver damage is characterized by a De Ritis ratio <1.0, whilst severe liver diseases are associated with values $>1.0(39,40)$. According to the study by Zinellu et al (39), the median De Ritis ratio was 1.33, similar to that reported by Yazar et al in their cohort (38). Furthermore, according to the study by Qin et al (41), the cut-off value of the De Ritis ratio was found to be 1.38; patients with a De Ritis ratio $>1.38$ upon admission have a significantly poor survival (41). With the hepatic proportion of the AST:ALT ratio of 2.5:1, the hepatocyte turnover may result in a much higher amount of AST in serum compared to ALT. AST has a shorter half-life of $18 \mathrm{~h}$ compared with ALT, which has a half-life of $36 \mathrm{~h}$. Hence, the serum levels of AST and ALT are fairly similar in healthy individuals. AST is present in the cytoplasm and mitochondria, whereas ALT is present only in the cytoplasm, although at a much higher concentration than AST (42). In the present study, the De Ritis ratio exhibited a positive correlation with the total bilirubin, D-dimer and AST levels, and a negative correlation with the hemoglobin, total WBC, serum albumin and ALT levels (Table IV).

In the present study, there was a significant increase in total bilirubin levels between mild and moderate cases ( $\mathrm{P}=0.04$; Table I). The serum albumin levels however, did not exhibit a statistically significant difference between the groups ( $\mathrm{P}=0.09$; Table II). Albumin is considered to be a negative acute phase reactant with low blood levels in acute inflammation and is inversely associated with the extent of systemic inflammation. In COVID-19 infection, an inverse association has been found between serum albumin levels and the severity of the disease (43). In the present study, in severe cases, there was a significant increase in LDH levels, but not in AST levels. This may be due to rhabdomyolysis due to direct viral invasion into myocytes. In addition, it may be due to the viral toxin-induced injury deposition of viral antigen antibody immune complexes or due to T-cell mediated injury as part of the manifestation of CSS $(11,44)$. Decreased WBC counts and platelets, along with an increased NLR and ferritin levels helps in assessing prognosis and appropriate treatment according to the severity of COVID-19 (45).

The present study was a retrospective study. Hence, information on IL-6, prothrombin, partial thromboplastin time, etc. could not be obtained. Moreover, this was a single-center study; thus, the population distribution and the severity of the patients who presented to the hospital would have played a major role in the findings of the study. The number of patients in the moderate and severe groups was less, which again may have compromised the results regarding liver function.

COVID-19 disease is characterized by extensive inflammation and various tissue involvement according to the severity of the disease. Hence, increased levels of inflammatory markers, as well as markers of organ involvement, such as the liver indicate that the disease has progressed from moderate to severe disease. The present study indicated there was widespread inflammation, with alterations in inflammatory markers, such as leukocytosis, neutrophilia, lymphopenia, and an increased NLR, as well as also increased levels of inflammatory markers, such as CRP, ferritin, LDH and D-dimer levels. Organ (liver) involvement was evidenced by increased levels of transaminases and increased total bilirubin. Furthermore, a correlation was found between the NLR and total WBC count, monocytes, albumin, CRP, ALT, ferritin, D-dimer and LDH levels. 
Such type of correlation was not observed for the De Ritis ratio. Hence, NLR and AST may be be better indicators of liver involvement than the De Ritis ratio in predicting systemic inflammation associated with liver injury or inflammation in patients with COVID-19.

\section{Acknowledgements}

Not applicable.

\section{Funding}

No funding was received.

\section{Availability of data and materials}

The datasets used and/or analyzed during the current study are available from the corresponding author on reasonable request.

\section{Authors' contributions}

SM was involved in the conception of the study. SM and TK were involved in data collection and compilation. MKK and SS were involved in the analysis of the data. SS and SM were involved in the preparation of the first draft of the article. SM and SS confirm the authenticity of all the raw data. All authors have read and approved the final version of the article.

\section{Ethics approval and consent to participate}

The present study was approved by the Institutional Ethics Committee of Sri Ramachandra Institute of Higher Education and Research, with a waiver of informed consent of patients, as permitted by the national regulatory body (IEC-NI/20/AUG/75/49, dated 08-08-2020). Patient identification was de-identified reversibly for the purpose of the analysis.

\section{Patient consent for publication}

Not applicable.

\section{Competing interests}

The authors declare that they have no competing interests.

\section{References}

1. Kenneth Oh: Aberrant cytokine activity in the host immune response to COVID-19 leads to cytokine release syndrome Bio-Rad Bulletin_7335; May, 2020. https://www.bio-rad.com/ webroot/web/pdf/lsr/literature/Bulletin_7335.pdf.

2. Hanif M, Haider MA, Xi Q, Ali MJ and Ahmed MU: A review of the risk factors associated with poor outcomes in patients with coronavirus disease 2019. Cureus 12: e10350, 2020.

3. Government of India Ministry of Health and Family Welfare: Clinical Management Protocol for COVID-19 (adults). https://www.mohfw.gov.in/pdf/UpdatedDetailedClinicalManagementProtocolforCOVID19adultsdated24052021.pdf. Accessed, July 27, 2021.

4. Bernheim A, Mei X, Huang M, Yang Y, Fayad ZA, Zhang N, Diao K, Lin B, Zhu X, Li K, et al: Chest CT findings in coronavirus disease-19 (COVID-19): Relationship to duration of infection. Radiology 295: 200463, 2020.
5. Falasca K, Ucciferri C, Brandimarte A, Auricchio A, Pontolillo M, Caiazzo L and Vecchiet J: Clinical characteristics and cardiovascular implications of the dead patients for COVID-19. Eur J Inflam 19, 2021.

6. Ali AHM, Mohamed SOO, Elkhidir IHE, Elbathani MEH, Ibrahim AAH, Elhassan ABE, Salman MST, Elhassan MAM, Elnil M and Abuzied AIH: The association of lymphocyte count, CRP, D-Dimer, and LDH with severe coronavirus disease 2019 (COVID-19): A meta-analysis. Sudan J Med Sci 15: 9-23, 2020.

7. Büyükaydın B: The relationship of hemogram and inflammatory biomarkers to lenght of stay in hospital and clinical course in patients with COVID-19. Bezm Sci 8: 7-14, 2020.

8. Moutchia J, Pokharel P, Kerri A, McGaw K, Uchai S, Nji M and Goodman M: Clinical laboratory parameters associated with severe or critical novel coronavirus disease 2019 (COVID-19): A systematic review and meta-analysis. PLoS One 15: e0239802, 2020.

9. Zhao Y, Nie HX, Hu K, Wu XJ, Zhang YT, Wang MM, Wang T, Zheng SC, Li XC and Zeng SL: Abnormal immunity of nonsurvivors with COVID-19: predictors for mortality. Infect Dis Poverty 9: 108, 2020.

10. Pourabdollah Toutkaboni M, Askari E, Khalili N, Tabarsi P, Jamaati H, Velayati AA, Dorudinia A, Rezaei M, Nadji SA, Mohamadnia A and Khalili N: Demographics, laboratory parameters and outcomes of 1061 patients with coronavirus disease 2019: A report from Tehran, Iran. New Microbe and New Infect 38: 100777, 2020 .

11. Kordzadeh-Kermani E, Khalili H and Karimzadeh I: Pathogenesis, clinical manifestations and complications of COVID-19. Future Microbiol 15: 1287-1305, 2020.

12. Thompson S, Bohn MK, Mancini N, Loh TP, Wang CB Grimmler M, Yuen KY, Mueller R, Koch D, Sethi S, et al: Horvath and the IFCC taskforce on COVID-19. IFCC interim guidelines on biochemical/hematological monitoring of COVID-19 patients. Clin Chem Lab Med 58: 2009-2016, 2020.

13. Danwang C, Endomba FT, Nkeck JR, Wouna DLA, Robert A and Noubiap JJ: A meta-analysis of potential biomarkers associated with severity of coronavirus disease 2019 (COVID-19). Biomark Res 8: 37, 2020.

14. Ucciferri $\mathrm{C}$, Vecchiet $\mathrm{J}$ and Falasca $\mathrm{K}$ : Role of monoclonal antibody drugs in the treatment of COVID-19. World J Clin Cases 8: 4280-4285, 2020.

15. Zenga F, Huangc Y, Guoa Y, Yina M, Chena X, Xiaod L and Deng G: Association of inflammatory markers with the severity of COVID-19: A meta-analysis. Int J Infect Dis 96: 467-474, 2020.

16. Jurado A, Martín MC, Abad-Molina C, Orduña A, Martínez A, Ocaña E, Yarce O, Navas AM, Trujillo A, Fernández L, et al: COVID-19: Age, interleukin-6, C-reactive protein, and lymphocytes as key clues from a multicentre retrospective study. Immun Ageing 17: 22, 2020.

17. Khourssaji M, Chapelle V, Evenepoel A, Belkhir L, Yombi JC, van Dievoet MA, Saussoy P, Coche E, Fillée C, Constantinescu SN, et al: A biological profile for diagnosis and outcome of COVID-19 patients. Clin Chem Lab Med 58: 2141-2150, 2020.

18. Marfia G, Navone S, Guarnaccia L, Campanella R, Mondoni M, Locatelli M, Barassi A, Fontana L, Palumbo F, Garzia E, et al: Decreased serum level of sphingosine-1-phosphate: A novel predictor of clinical severity in COVID-19. EMBO Mol Med 13: e13424, 2021.

19. Hu H, Du H, Li J, Wang Y, Xu X, Wang C, Zhang Y, Zhang G, Zhao Y, Kang W and Lian J: Early prediction and identification for severe patients during the pandemic of COVID-19: A severe COVID-19 risk model constructed by multivariate logistic regression analysis. J Glob Health 10: 020510, 2020.

20. Mirmohammadi S, Kianmehr A, Arefi M and Mahrooz A: Biochemical parameters and pathogenesis of SARS-CoV-2 infection in vital organs: COVID-19 outbreak in Iran. New Microbes New Infect 38: 100792, 2020.

21. Alhenc-Gelas F and Drueke TB: Blockade of SARS-CoV-2 infection by recombinant soluble ACE2. Kidney Int 97: 1091-1093, 2020.

22. Zhang L, Peng Y, Zheng Q, Jiang L, Tang S and Chen P: Retrospective analysis of clinical characteristics and laboratory results of COVID-19 patients. European Journal of Inflammation. January 2021. doi:10.1177/20587392211011919.

23. Kho J, Ioannou A, Van den Abbeele K, Mandal AKJ and Missouris CG: Pulmonary embolism in COVID-19: Clinical characteristics and cardiac implications. Am J Emerg Med 38: 2142-2146, 2020 . 
24. Farooqi F, Dhawan N, Morgan R, Dinh J, Nedd K and Yatzkan G: Treatment of severe COVID-19 with Tocilizumab mitigates cytokine storm and averts mechanical ventilation during acute respiratory distress: A case report and literature review. Trop Med Infect Dis 5: 112, 2020.

25. Guillén L, Padilla S, Fernández M, Agulló V, García JA, Telenti G, García-Abellán J, Botella Á, Gutiérrez F and Masiá M: Preemptive interleukin-6 blockade in patients with COVID-19. Sci Rep 10: 16826, 2020.

26. Cavalli G, Farina N, Campochiaro C, De Luca G, Della-Torre E, Tomelleri A and Dagna L: Repurposing of biologic and targeted synthetic Anti-Rheumatic drugs in COVID-19 and Hyper-Inflammation: A comprehensive review of available and emerging evidence at the Peak of the Pandemic. Front Pharmacol 11: 598308, 2020.

27. Li H, Liu L, Zhang D, Xu J, Dai H, Tang N, Su X and Cao B: SARS-CoV-2 and viral sepsis: Observations and hypotheses Lancet 395: 1517-1520, 2020.

28. Ponti G, Maccaferri M, Ruini C, Tomasi A and Ozben T: Biomarkers associated with COVID-19 disease progression. Crit Rev Clin Lab Sci 57: 389-399, 2020.

29. Huang I, Pranata R, Lim MA, Oehadian A and Alisjahbana B: C-reactive protein, procalcitonin, D-dimer, and ferritin in severe coronavirus disease-2019: A meta-analysis. Ther Adv Respir Dis 14: 1753466620937175, 2020.

30. Weidmann MD, Ofori K and Rai AJ: Laboratory Biomarkers in the management of patients with COVID-19. Am J Clin Pathol 155: 333-342, 2021.

31. Gris JC, Perez-Martin A, Quéré I and Sotto A: COVID-19 associated coagulopathy: The crowning glory of thrombo-inflammation concept. Anaesth Crit Care Pain Med 39: 381-382, 2020.

32. Yazdanpanah F, Hamblin MR and Rezaei N: The immune system and COVID-19: Friend or foe? Life Sci 256: 117900, 2020

33. Zhang L and Guo H: Biomarkers of COVID-19 and technologies to combat SARS-CoV-2. Adv Biomark Sci Technol 2: 1-23, 2020

34. South AM,BradyTMandFlynnJT:ACE2(Angiotensin-Converting Enzyme 2), COVID-19, and ACE Inhibitor and Ang II (Angiotensin II) Receptor blocker use during the Pandemic: The Pediatric perspective. Hypertension 76: 16-22, 2020.

35. Gong J, Ou J, Qiu X, Jie Y, Chen Y, Yuan L, Cao J, Tan M, Xu W, Zheng F, et al: A tool for early prediction of severe Coronavirus disease 2019 (COVID-19): A multicenter study using the risk Nomogram in Wuhan and Guangdong, China. Clin Infect Dis 71: $833-840,2020$
36. Adhikari IP, Tiwari R and Bala R: Estimation of the De Ritis ratio in the cases of chronic alcoholic liver disease attending OPD of a tertiary health care level institute of Kanpur, UP. PARIPEX-Indian J Res 7: 219-221, 2018.

37. Ghahramani S, Tabrizi R, Lankarani KB, Kashani SMA, Rezaei S, Zeidi N, Akbari M, Heydari ST, Akbari H, Nowrouzi-Sohrabi P and Ahmadizar F: Laboratory features of severe vs. non-severe COVID-19 patients in Asian populations: A systematic review and meta-analysis. Eur J Med Res 25: 30, 2020.

38. Yazar H, Kayacan Y and Ozdin M: De Ritis ratio and biochemical parameters in COVID-19 patients. Arch Physiol Biochem: 1-5, 2020 (Epub ahead of print).

39. Zinellu A, Arru F, De Vito A, Sassu A, Valdes G, Scano V, Zinellu E, Perra R, Madeddu G, Carru C, et al: The De Ritis ratio as prognostic biomarker of in-hospital mortality in COVID-19 patients. Eur J Clin Invest 51: e13427, 2021.

40. Parmar KS, Singh GK, Gupta GP, Pathak T and Nayak S: Evaluation of De Ritis ratio in liver-associated diseases. Int J Med Sci Public Health 5: 1783-1788, 2016.

41. Qin C, Wei Y, Lyu X, Zhao B, Feng Y, Li T, Cao H, Yang X, Zhou X, Wang W, et al: High aspartate aminotransferase to alanine aminotransferase ratio on admission as risk factor for poor prognosis in COVID-19 patients. Sci Rep 10: 16496 2020.

42. Botros M and Sikaris KA: The de ritis ratio: The test of time. Clin Biochem Rev 34: 117-130, 2013.

43. Varim C, Yaylaci S, Demirci T, Kaya T, Nalbant A, Dheir H, Senocak D, Kurt R, Cengiz H and Karacaer C: Neutrophil count to albumin ratio as a new predictor of mortality in patients with COVID-19 infection. Rev Assoc Med Bras (1992) 66 (Suppl 2): S77-S81, 2020.

44. Ucciferri C, Caiazzo L, Di Nicola M, Borrelli P, Pontolillo M, Auricchio A, Vecchiet J and Falasca K: Parameters associated with diagnosis of COVID-19 in emergency department. Immun Inflamm Dis 9: 851-861, 2021.

45. Kabak M, Çil B and Hocanlı I: Relationship between leukocyte, neutrophil, lymphocyte, platelet counts, and neutrophil to lymphocyte ratio and polymerase chain reaction positivity. Int Immunopharmacol 93: 107390, 2021.

This work is licensed under a Creative Commons Attribution-NonCommercial-NoDerivatives 4.0 International (CC BY-NC-ND 4.0) License. 